\title{
Estimating spectral heart rate variability (HRV) features with missing RR-interval data
}

\author{
Md. Surat-E-Mostafa \\ Center for Machine Vision and Signal Analysis \\ University of Oulu \\ Oulu, Finland \\ surat153@gmail.com \\ Metali Rani Datta \\ Lecturer \\ European University of Bangladesh \\ Dhaka, Bangladesh \\ metalidatta02@gmail.com
}

\author{
Md. Al Faisal Reza \\ Lecturer \\ European University of Bangladesh \\ Dhaka, Bangladesh \\ mafrsabuj@gmail.com \\ Rawshon Ara Rupa \\ Student \\ M Abdur Rahim Medical College \\ Dinajpur, Bangladesh \\ rawshonrupa124@gmail.com
}

\author{
Sakib Mostafa \\ Student \\ University of Rajshahi \\ Rajshahi, Bangladesh \\ sakib.iba6618@gmail.com
}

\begin{abstract}
Physiological signals, ECG signal, have been widely used for diagnosis, disease identification and nowadays for selfmonitoring. Missing data represents the problem in spectral analysis. This study focuses on the HRV power spectral analysis in frequency-domain using three methods with simulated missing data in real RR-interval tachograms. Actual missing ECG data are collected from the unconstrained measurement. Parametric, Non-parametric and uneven sampling approach were used for calculating the power spectral density (PSD), and cubic spline interpolation method was used for the non-parametric method. Based on this studies outcome, the effect of missing RR-interval data and optimal method was observed through the simulated real RR-interval tachograms for missing data. About 0 to 6 percentage data were removed according to the exponential Poisson distribution from the real RR-interval data for normal sinus rhythm, atrial fibrillation, tachycardia and bradycardia patient which data obtained from MIT-BIH Arrhythmia database to simulate real-world packet loss. For this analysis, 5 min duration data were used in all and 1000 Monte Carlo runs is performed for certain percentage missing data. PSD corresponding each frequency component was estimated as the frequency-domain parameters in each run and error power percentage based on each element difference between with and without the missing data duration were calculated.

Index Terms-Electrocardiogram, missing data, atrial fibrillation, tachycardia, bradycardia, power spectral density.
\end{abstract}

\section{INTRODUCTION}

The analysis of heart rate variability is a process to study the automatic nervous system (ANS) and the evaluation of clinical cardiac risk. To observe the heart rate variability is performed in the time and frequency domain techniques among the several proposed techniques [1]. Spectral analysis was first introduced in astronomical time-series analysis for unevenly sampled data in 1969. Besides, Task Force of the European
Society of Cardiology in the North American described the HRV estimation methods in 1996 [2]. After that, the US Food and Drug Administration (FDA) approved the medical devices for calculating the HRV unless the results are not helped to make the specific medical diagnosis. Furthermore, in the last few years frequency domain technique spread out to find out the fluctuation patterns on the RR interval in time series, and it helps in clinical diagnosis and prognosis [3]. Most of the HRV measurement studies predict the risk of mortality (prognosis). But there are only a several studies which focused on diagnosis purpose [1]. The HRV data is extracted from electrocardiogram (ECG) records. The central spike of ECG record is QRS complex seen in ECG. Since R peaks in QRS complexes represent the ventricular contractions, the beats instants are taken at these points. The beat-to-beat intervals are calculated by measuring the time distance between $R$ waves. Therefore, the term "beat-to-beat interval" known as an RR-interval which is an unevenly sampled data [1]. Clayton et al. [4] described that FFT and AR methods can estimate the comparable low-frequency (LF) and high-frequency (HF) metrics for linearly sampled data (the power between 0.04 to $0.15 \mathrm{~Hz}$ and 0.15 to $0.4 \mathrm{~Hz}$, respectively ) [5]. To handle with unevenly sampled data issue, RR-interval data needed to be interpolated by the cubic spline and sampled at $4 \mathrm{~Hz}$ for Fast Fourier Transform (FFT) and Autoregressive Model (AR) because both techniques require evenly sampled data [3]. Besides, it is essential to remove the non-sinus (ectopic) beats from unevenly sampled RR tachogram because ectopic beats introduce further resampling problems and increase the variance of the interbeat intervals [5]. The ectopic beat should be removed from RR-tachograms for HRV analysis because variability in the RR-intervals may occur due to the ectopic beat. Usually, ectopic beat often occurs earlier and sometimes 
later than normal rhythms to happen. These unwanted short RR-intervals make higher than standard frequency component. As a result, HRV measurement can be distorted from the actual measurement [2].

Birkett et al. [6] computed HRV spectra for congestive heart failure patients in two ways: firstly, ectopic beats were replaced by linear and cubic spline interpolation [7]. Secondly, ectopic beats were discarded. They concluded that high Frequency (HF) components were unaffected but low-frequency components (LF) was much higher using either interpolation method. In 1994 Lippman et al. [8] observed the linear, cubic, nonlinear prediction interpolation and the null case. After that, they summarized that ectopic correction should take into account for HRV analysis. Their finding was that linear, and cubic interpolation can interfere in power spectral estimations.

Furthermore, Lomb-Scargle (LS) method shows better performance to estimate the PSD [9], [10], [11] and it is analyzed by Moody et al. [12], [13]. Their result shows that PSD estimation is more accurate for typical RR-interval tachograms in LS method compared to the FFT method. Because LS method does not need any resample and detrend RR data for estimating the PSD. [6], [10].

The aim of this study is to compare between different spectral estimation methods for missing data and exploring the optimal method when applied to HRV frequency domain analysis through Monte Carlo simulation using the RRinterval model. In this simulation, several spectral estimation techniques are used for PSD estimation. Besides, the longterm normal, atrial fibrillation (AF), tachycardia and bradycardia patient's RR-interval tachograms are considered for the simulation of missing data for observing the missing data effect. The simulated gap generated based on the Poisson exponential distribution because actual missing data follow the Poisson exponential distribution method which obtained from unconstrained ECG measurements.

\section{Spectral Methods for Estimating HRV PARAMETERS}

Spectral-based methods aim to describe the distribution of power of a signal over frequency. The statistical properties of biosignals vary in time. That is why it is called nonstationary signals. To estimate the spectral density of constructed signals, we have used frequency analysis methods. For this purpose, we have used Fast Fourier transform (FFT) method (Welch's Power Spectral Density), Auto-Regressive (AR) method (Burg's Power Spectral Density) and LombScargle (LS) method among various method.

Power spectral density (PSD) can be calculated by the parametric and nonparametric method. Each of this method has different advantages. Fast Fourier Transform (FFT) is a non-parametric method for determining the PSD. It has a high processing speed and a simple algorithm. This method requires evenly spaced data for estimating the PSD. But RRinterval data is unevenly spaced sampled data. That is why these method has been suggested to interpolate the unevenly sampled RR- interval data. In this study, we interpolated the
RR-intervals series using cubic spline at sampled at $4 \mathrm{~Hz}$ [14]. To estimate the actual power spectrum is not a consistent measure because it has leakage problems and bias. So it can spoil the spectrum. As an alternative to removing those problems, Welch's method comes forward [14]. Based on this, we used Welch's method to estimate the PSD in this research. Welch's method is used for estimating the power of a signal at different frequencies. This method involves sectioning the signal, taking modified periodograms of these sections, and averaging these modified periodograms [15]. Based on the periodogram spectrum estimation, the method converts a signal from the time domain to the frequency domain. Welch's method is also called the averaging method and weighted overlapped segment averaging (WOSA) method [16]. In our research, we used the Hanning window for each segment. Its length is equal to the sampling frequency of the signal. And we consider the 50 percent overlap between each segment.

The spectral analysis describes the power of signal over frequency. A time-frequency representation used in this study is Brug's-based Autoregressive (AR) method. AR method is the parametric method. In the parametric method, there is an all-pole linear filter which discusses the signal's spectral components. AR method performs efficiently for large PSD at certain frequencies. Besides, when the signal data is relatively short, the AR method generate the good result compared to the non-parametric methods. Moreover, AR method generates smoother PSD than non-parametric methods. Burg's method is developed for estimating the AR parameters that are based on forward and backward prediction errors. In this study, Burg's method used for AR spectral estimation that minimizes the forward and backward prediction error by constraining the AR parameters according to the Levinson-Durbin recursion [17]. Levinson-Durbin recursion predicts the linearity of an all-pole IIR filter with deterministic autocorrelation sequence. In this analysis, model order 11th is used with sampling frequency $4 \mathrm{~Hz}$. The model order indicates the number of poles of the AR model. The burg's method shows the better result for estimation of short records data and better resolution of closely spaced sinusoids with low noise. Neophytos.N. [18] described that burg's method shows the small divergence of the PSD estimation from the true value. On the other hand, burg's method accuracy is decreasing for high model orders, more extended data and higher signal-to-noise ratios. Finally, this method usually behaves somewhere between the LS method and the Yule-Walker method [18].

The Lomb-Scargle method is used for performing the spectral analysis on missing sample data. The Lomb-scargle algorithm is well detecting and characterizing periodicity in missing sampled time-series. In this research, we used the lomb-scargle algorithm for calculating the PSD in missing sampled time-series. For classical methods, we can not estimate PSD directly for missing sampled data without resampling. This resampling can make artifacts in the estimated spectrum. On the other hand, for the Lomb-scargle method, we can calculate the spectrum directly for missing sampled data without any resampling or interpolation. And we can avoid 
spectrum distortion in the Lomb-scargle method [19]. In the Lomb method, PSD estimates based on the minimization of the squared differences between the signal and the basis function of the transform.

\section{MISSING DATA HANDLING}

In this study, we used the 21 patients real-time collected ECG data for analysis. The real time data were received by the wireless device with blue tooth connection. After the investigation, we have observed that there are the missing data due to blue tooth packet loss in real-world. Then, we have found the missing data duration percentage and nonmissing data duration percentage from each patient whole recorded data. Finally, we have drawn the histogram based on the missing and non-missing data distribution of real recorded data. And we observed that most of the patient missing data distribution follow the Poisson distribution. For this reason, in this analysis, we used the exponential Poisson distribution method to generate the simulated missing data using the PhysioNet database. For producing the missing data we use the exponential distribution for inter-event intervals as the number of events is Poisson distributed in the following way:

The Poisson distribution with probability mass function is:

$$
P(X)=\frac{e^{-\mu} \mu^{x}}{x !}
$$

where $\mathrm{x}=0,1,2, \ldots$, and $\mu$ is the mean distribution and $\mathrm{e}$ is the exponential. Here, the variance of this distribution is equal to the mean distribution [20]. On the other hand , exponential distribution is a continuous distribution with probability density function,

$$
f(t)=\lambda e^{-\lambda t}
$$

where $t \geq 0$ and the parameter $\lambda>0$. In this distribution, the mean and standard deviation are both equal to $\frac{1}{\lambda}$ [20]. So the cumulative exponential distribution is,

$$
F(t)=\int_{0}^{\infty} \lambda e^{-\lambda t} d t=1-e^{-\lambda t}
$$

\section{REAL MISSING DATA}

In our study, we used real data for investigating the effects of the real missing RR-interval data on HRV analysis and finding the optimal spectral method with missing data. In the real collected data, there are 21 patients about 6 hours recorded ECG data with motion artifact and missing parts which known as $\mathrm{NaN}$ in datasets. All subjects were cardiac disease patients. But most of the patients was arrested by atrial fibrillation, tachycardia and bradycardia heart diseases. RR-interval data were extracted by using the R-peak detection algorithm from the original ECG data. But motion artifacts are dominant in real ECG; hence, it makes the complexity to detect R-peaks correctly from real datasets. For this reason, we calculated the non-missing data part with missing data part from real datasets. Figure 1 provides the missing data and non-missing data characteristics of real data sets which have been figured out using the Poisson distribution parameters. After that, we calculated the consecutive missing run length from those 21 patients datasets. The missing run length distribution sample in the datasets is showed in figure 2. Besides, figure 2 presents the simulated gap length distribution based on the real missing data length characteristic. This missing data length in real datasets follows the exponential poison distribution.

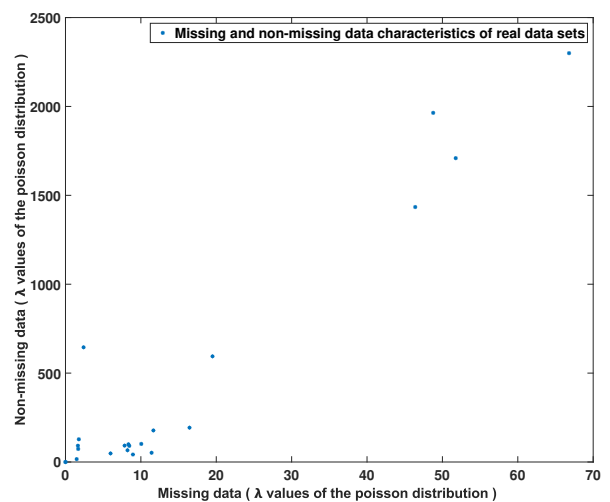

Fig. 1. Missing data Vs. Non-missing data characteristics of real datasets estimating using the Poisson distribution parameters.

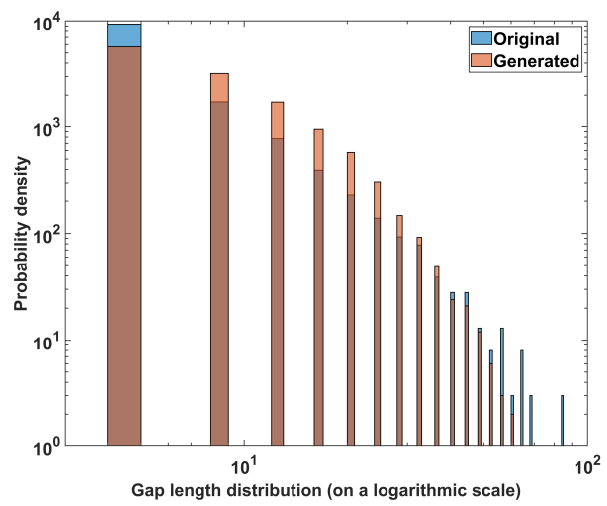

Fig. 2. Log-normal consecutive gap length distribution of real sample data and simulated gap length distribution.

\section{Simulation of The Missing DATA}

For this analysis, the long-term RR-interval tachograms data were collected from the MIT-BIH arrhythmia database (https://www.physionet.org/physiobank/database/mitdb/).There are 48 half-hour excerpts of two-channel ambulatory ECG recordings in the MIT-BIH Arrhythmia Database. After studying the BIH Arrhythmia Laboratory, 47 subjects obtained from 48 subjects. Twenty-three subjects from 47 were selected at random from a set of 4000 24-hour ambulatory ECG recordings from a mixed population. And the rest of the 25 subjects were chosen from the same set but clinically significant arrhythmias. Besides, the ECGs were digitized at 360 samples per second in every channel with 11-bit resolution and $10 \mathrm{mV}$ range. Moreover, two or more cardiologist annotated each record separately. And their 
disagreements were resolved using the computer-readable reference annotations for each beat. In this study, we used selectively extracted 5 minutes of ECG data including normal sinus rhythm, AF, tachycardia, and bradycardia patients for HRV analysis using previously mention three spectral based methods.

For the simulation, the data were removed by randomly from the RR-interval data. And randomly missing data duration remained between about 0 to 6 percentage based on the real missing data. Applying 1000 Monte Carlo runs using the MATLAB program, the HRV spectral parameters were estimated. In this study, the relative errors (REs) was used to observe the effect of missing data on HRV parameters in those three methods. The relative errors (REs) compared the missing data parameters with the original data parameters which have no missing data. The HRV parameters from the missing data is $\left[X_{1}, X_{2}, \cdots, X_{n}\right]$ where $n=1000$ and the $X_{o}$ rigin represents the parameter value from the non-missing data. Then, relative errors are calculated as,

$$
\operatorname{REs}(\%)=\frac{X_{\text {origin }}-X_{k}}{X_{\text {origin }}} \cdot 100
$$

Here $K$ represents $1,2, \cdots, n$ [21], [22].

\section{Simulation RESUlts}

Figure 3 shows the error power percentage between nonmissing data spectrum and missing data spectrum for normal patient based on the duration of missing data via FFT, AR, LS-based method respectively in sub-figure 1, 2 and 3 using box plot. The missing data duration axis expresses as a bin in which each bin contains 200 samples among 1000 Monte Carlo simulation samples. In this figure, the error power percentage for each HRV parameter was altered by different spectral estimation methods. In the sub-figure 1 of figure 3, it has been clear that median error remains almost same up to 3.06 percentage of missing data but median error increases gradually when missing data percentage goes above 3.06 for FFT method.

The same trend is followed in sub-figure 2 for AR method. In this case, the median error rises when the missing data percentage cross the bin2, amounting 2.59 percentage missing data. On the contrary, in sub-figure 3 for LS method, as the percentage of missing data increases, the percentage of median error power remains almost the same. Overall, in the LS method, there is less effect for missing data percentage compared to the FFT and AR method and number of outliers are fewer also. From this figure, it is clearly noticed that the error power percentage shows less amount between three estimates with LS for normal patient data in term of the missing data percentage.

After that, figure 4 represents the error power percentage between the non-missing data spectrum and missing data spectrum for AF patient via previously described three methods based on the duration of missing data. In sub-figure 1 and 2 , the median error is almost the same till 2.59 percentage of missing data for both cases namely FFT and AR method.
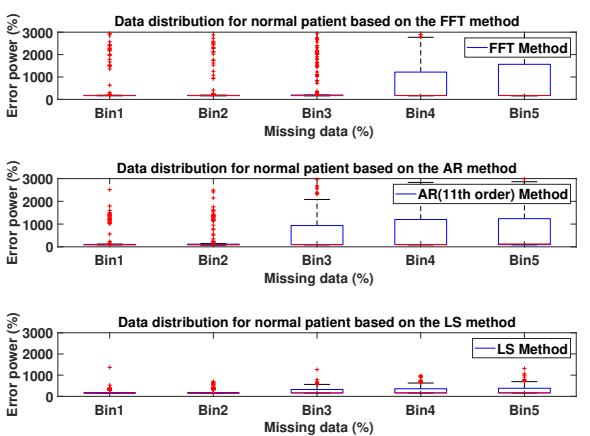

Fig. 3. Boxplot of the subfigure 1,2 and 3 estimated the error power percentage based on the missing data percentage for the normal patient via FFT, AR, and LS method respectively using 1000 times Monte Carlo simulated data. First quartile, median, and third quartile values are portrayed as the bottom, middle and top horizontal line of the boxes. Whiskers are used to represent the most extreme values. And outliers were displayed as crosses. The error power percentage was calculated by the equation 4. Each Bin contains missing data percentage respectively $\operatorname{Bin} 1(0.20$ to 2.10$)$, $\operatorname{Bin} 2(2.11$ to 2.59$)$, $\operatorname{Bin} 3(2.60$ to 3.06$)$, Bin4(3.07 to 3.59) and $\operatorname{Bin} 5(3.60$ to 5.60)
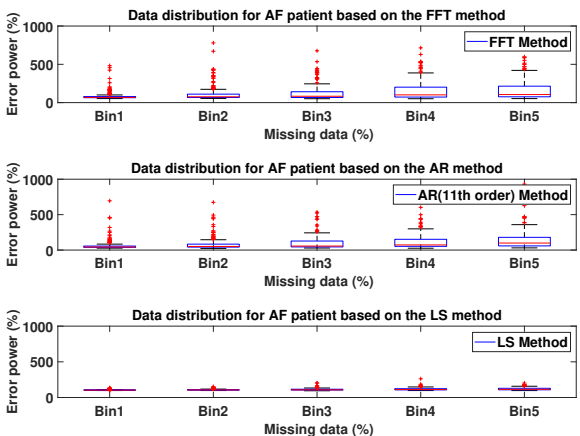

Fig. 4. Boxplot of the subfigure 1,2 and 3 estimated the error power percentage based on the missing data percentage for the AF patient via FFT, AR, and LS method respectively using 1000 times Monte Carlo simulated data. First quartile, median, and third quartile values are portrayed as the bottom, middle and top horizontal line of the boxes. Whiskers are used to represent the most extreme values. And outliers were displayed as crosses. The error power percentage was calculated by the equation 4. Each Bin contains missing data percentage respectively $\operatorname{Bin} 1(0.20$ to 2.10$)$, $\operatorname{Bin} 2(2.11$ to 2.59$)$, $\operatorname{Bin} 3(2.60$ to 3.06$)$, Bin4(3.07 to 3.59) and $\operatorname{Bin} 5(3.60$ to 5.60).

Thereafter, in both instances median error increases slowly when the missing data percentage goes above 2.59 percentage, whereas median error remains almost constant for LS method in term of the missing data percentage in sub-figure 3. Besides, the number of outliers is less in LS method compared to the other two methods namely FFT and AR method.

Furthermore, figure 5 indicates the error power percentage between the non-missing data spectrum and missing data spectrum for tachycardia patient through previously described three methods based on the duration of missing data. From sub-figure 1 and 2, median error gradually climbs up as the increase of the missing data percentage for FFT and AR method. On the other hand, from sub-figure 3 the median error 
power keep almost constant up-to 3.06 percentage of missing data. After that, there is little bit change when missing data goes above 3.06 percentage. Besides, the number of outliers are fewer in LS method compared to the FFT and AR methods in term of the missing data percentage
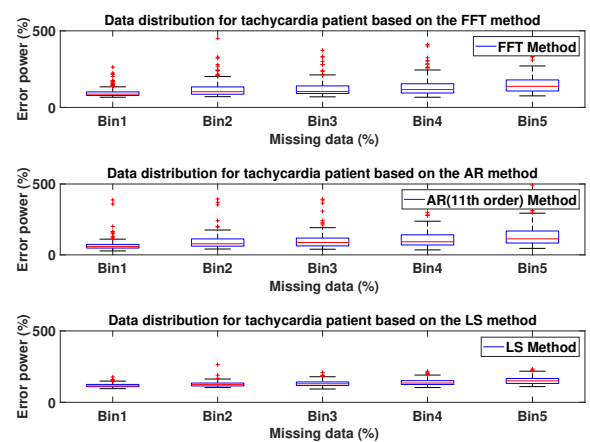

Fig. 5. Boxplot of the subfigure 1,2 and 3 estimated the error power percentage based on the missing data percentage for the tachycardia patient via FFT, AR, and LS method respectively using 1000 times Monte Carlo simulated data. First quartile, median, and third quartile values are portrayed as the bottom, middle and top horizontal line of the boxes. Whiskers are used to represent the most extreme values. And outliers were displayed as crosses. The error power percentage was calculated by equation 4 . Each Bin contains missing data percentage respectively $\operatorname{Bin} 1(0.20$ to 2.10$)$, $\operatorname{Bin} 2(2.11$ to 2.59 ), Bin3(2.60 to 3.06), Bin4(3.07 to 3.59) and Bin5(3.60 to 5.60).

Finally, figure 6 reveals the error power percentage between the non-missing data spectrum and missing data spectrum for bradycardia patient based on the duration of missing data. It is cleared from sub-figure 3 that the median error power lies more or less the same as the percentage of missing data increase although the median error power is higher compared to the other two methods. On the other hand, the median error power remains the same till 2.59 percentage of missing data for FFT and AR methods, showed in sub-figure 1 and 2. After that, the median error minimally increases when the missing data percentage goes above 2.59 percentage. Besides, the number of outliers are more in FFT and AR methods, whereas outlier's number are less in the LS method.

Now figure 7 shows the comparison between those three spectral-based method based on the estimated median value which extracted from the figures $3,4,5$ and 6 . In this figure, sub-figures $1,2,3$ and 4 represent the normal, AF, tachycardia and bradycardia patient result respectively. From all sub-figures, it is clearly visualized that the AR method shows the significantly lower median value corresponding the missing data percentage compared to the others two methods for normal, AF, tachycardia and bradycardia patient.

\section{CONCLUSION}

Our research focused on the comparison between three spectral methods performance based on the missing data percentage with normal and three different types of patients data and also observed the effect of missing data among those
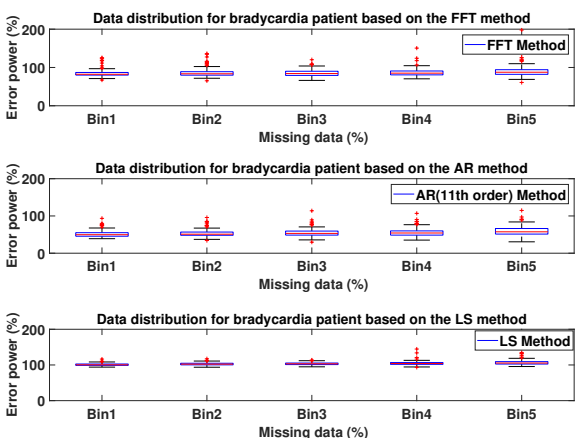

Fig. 6. Boxplot of the subfigure 1,2 and 3 estimated the error power percentage based on the missing data percentage for the bradycardia patient via FFT, AR, and LS method respectively using 1000 times Monte Carlo simulated data. First quartile, median, and third quartile values are portrayed as the bottom, middle and top horizontal line of the boxes. Whiskers are used to represent the most extreme values. And outliers were displayed as crosses. The error power percentage was calculated by the equation 4. Each Bin contains missing data percentage respectively $\operatorname{Bin} 1(0.20$ to 2.10$)$, $\operatorname{Bin} 2(2.11$ to 2.59$)$, $\operatorname{Bin} 3(2.60$ to 3.06), $\operatorname{Bin} 4(3.07$ to 3.59) and $\operatorname{Bin} 5(3.60$ to 5.60).

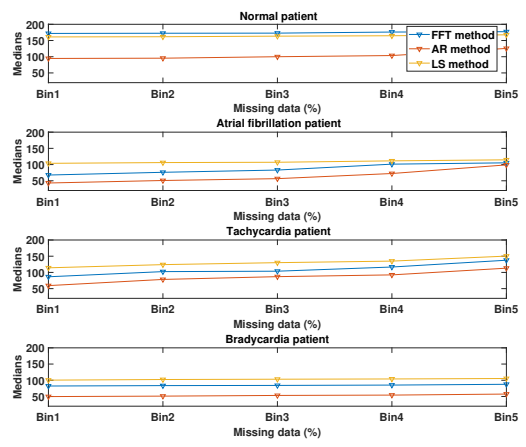

Fig. 7. This plot shows the comparison between FFT, AR and LS method based on the estimated median value of error power percentage for normal, $\mathrm{AF}$, tachycardia and bradycardia patient from figure $[3,4,5,6]$. In figure, the red line, blue line and yellow line represent the AR, FFT and LS method respectively. Each Bin contains missing data percentage respectively $\operatorname{Bin} 1(0.20$ to 2.10$), \operatorname{Bin} 2(2.11$ to 2.59$), \operatorname{Bin} 3(2.60$ to 3.06$), \operatorname{Bin} 4(3.07$ to 3.59$)$ and $\operatorname{Bin} 5(3.60$ to 5.60$)$.

three spectral methods. In this research, one subject has experimented for each normal, AF, tachycardia and bradycardia patient. We used 5 minutes of ECG data for each case and generated 1000 times Monte Carlo simulation for making the artificial gap in RR-interval tachograms. This research presents that the artificial gap generation from RR-interval tachograms and the calculation of HRV through FFT and AR methods generates significant errors due to the resampling step. The LS method performs to reduce these errors even in a large amount of missing data. By observing the result, it is cleared that LS method shows the lower variance estimate of the frequency components compared to the FFT and AR method for normal, $\mathrm{AF}$, tachycardia and bradycardia patient based on the missing data percentage. This lower variance estimation indicates that 
the LS method for calculating spectral HRV are more robust and accurate corresponding to the reference signal. On the other hand, AR method shows the better performance to determine the PSD with a certain percentage of missing data.In a future study, nonlinear HRV parameter will be observed with missing data for various diseases patients.

\section{REFERENCES}

[1] Y.Isler and M. Kuntalp, "Combining classical hrv indices with wavelet entropy measures improves to performance in diagnosing congestive heart failure," Computers in biology and medicine, vol. 37, pp. 1502$1510,2007$.

[2] G. D. Clifford and L. Tarassenko, "Quantifying errors in spectral estimates of hrv due to beat replacement and resampling," IEEEtransactions on biomedical engineering, vol. 52, pp. 630-638, 2005.

[3] D. Fonseca, A. Netto, R. Ferreira, and A. M. de Sa, "Lomb-scargle periodogram applied to heart rate variability study," in Biosignals and Biorobotics Conference (BRC), pp. 1-4, 2013.

[4] R. Clayton, S. Lord, J. McComb, and A. Murray, "Comparison of autoregressive and fourier transform based techniques for estimating rr interval spectra," in Computers in Cardiology 1997. IEEE, pp. 379-382, 1997.

[5] M. Malik, J. T. Bigger, A. J. Camm, R. E. Kleiger, A. Malliani, A. J. Moss, and P. J. Schwartz, "Heart rate variability: Standards of measurement, physiological interpretation, and clinical use," European heart journal, vol. 17, pp. 354-381, 1996.

[6] C. Birkett, M. Kienzle, and G. Myers, "Interpolation over ectopic beats increases low frequency power in heart rate variability spectra," in Computers in Cardiology 1991, Proceedings. IEEE, pp.257-259, 1991.

[7] L. Piegl, "On nurbs: a survey,' IEEE Computer Graphics and Applications," IEEE, vol. 11, pp. 55-71, 1991

[8] N. Lippman, K. M. Stein, and B. B. Lerman, "Comparison of methods for removal of ectopy in measurement of heart rate variability," American Journal of Physiology-Heart and Circulatory Physiology, vol. 267, pp. H411-H418, 1994.

[9] N. R. Lomb, "Least-squares frequency analysis of unequally spaced data," Astrophysics and space science, vol. 267, pp. 447-462, 1976.

[10] J. D. Scargle, “ Studies in astronomical time series analysis. ii-statistical aspects of spectral analysis of unevenly spaced data," The Astrophysical Journal, vol. 263, pp. 835-853, 1982.

[11] W. H. Press, S. A. Teukolsky, W. T. Vetterling, and B. P. Flannery, " Numerical recipes 3rd edition: The art of scientific computing," Cambridge university press, 2007

[12] G. B. Moody, "Spectral analysis of heart rate without resampling," in Computers in Cardiology 1993, Proceedings. IEEE, pp. 715-718, 1993.

[13] S. Abeysekera, U. R. Abeyratne, and S. Goh, "Spectral information changes in obtaining heart rate variability from tachometer rr interval signals," Critical Reviews in Biomedical Engineering, vol. 28, pp. 1-2, 2000.

[14] D. Fonseca, A. Netto, R. Ferreira, and A. M. de Sa, "Lomb-scargle periodogram applied to heart rate variability study, in Biosignals and Biorobotics Conference (BRC)," 2013 ISSNIP. IEEE, pp. 1-4, 2013.

[15] P. Welch, "The use of fast fourier transform for the estimation of power spectra: a method based on time averaging over short, modified periodograms," IEEE Transactions on audio and electroacoustics, vol. 15 , pp. 70-73, 1967.

[16] O. Solomon Jr, "Psd computations using welch's method," NASA STI/Recon Technical Report N, vol. 92, 1991.

[17] P. Brockwell and R. Dahlhaus, "Generalized levinson-durbin and burg algorithms," Journal of Econometrics, vol. 118, pp. 129-149, 2004.

[18] N.NeovTou, "Ecg event detection recognition using time-frequency analysis," Ph.D. dissertation, 2012.

[19] J. T. VanderPlas, "Power Spectral Density of Unevenly Sampled Heart Rate Data," The Astrophysical Journal Supplement Series, 236:16 (28pp), vol. 15, pp. 236-254, 2018.

[20] J. C. Cooper, "The poisson and exponential distributions," Mathematical Spectrum, vol. 37, pp. 123-125, 2005.

[21] K. K. Kim, J. S. Kim, Y. G. Lim, and K. S. Park, "The effect of missing rr-interval data on heart rate variability analysis in the frequency domain," Physiological measurement, vol. 30, pp. 1039, 2009.
[22] K. K. Kim, H. J. Baek, Y. G. Lim, and K. S. Park, “ Effect of missing RR-interval data on nonlinear heart rate variability analysis," Comput Methods Programs Biomed.2012 Jun;106(3):210-8, 2012. 